\title{
La escritura en el aula como instrumento de aprendizaje. Estudio en universidades*
}

\author{
Writing in the classroom as a learning tool. \\ Research at universities
}

A escritura na sala de aula como uma ferramenta de aprendizagem. Estudo em universidades

Recibido el 15 de octubre de 2014. Aceptado el 17 de enero de 2015

\author{
Camilo Giraldo Giraldo** \\ Colombia
}

\section{Resumen}

, Para citar este artículo:

Giraldo Giraldo, Camilo (2015).

La escritura en el aula como instrumento de aprendizaje.

Estudio en universidades. Ánfora,

22 (38), 39-58. Universidad

Autónoma de Manizales. ISSN

0121-6538
Objetivo: establecer si en las aulas universitarias de Manizales se hace uso intencional de la escritura como instrumento de enseñanza-aprendizaje e identificar estrategias de escritura aplicadas en el aula y las posibles orientaciones curriculares al respecto. Metodología: se aplicó encuesta con preguntas cerradas y abiertas y entrevista abierta a 200 profesores de cinco universidades de Manizales. Con ello, se buscó la verificación de dos hipótesis: a) en el aula universitaria no se usa, intencionalmente, la escritura como instrumento para la enseñanza-aprendizaje y b) los

\footnotetext{
* Este artículo hace parte del proyecto de investigación en "Comunicación escrita" que adelanta el autor como integrante del grupo de Investigaciones de la Comunicación de la Universidad de Manizales, Colombia.

** Magíster en Filosofía. Profesor e investigador de la Universidad de Manizales. Correo electrónico: cgiraldogiraldo@gmail.com
} 
docentes consideran que la enseñanza de la escritura se debe dejar a las materias de lectoescritura. Resultados: se corroboró la primera hipótesis con el 99\% de las respuestas. De los 200 docentes participantes, 198 respondieron que no usan la escritura en el aula para apuntalar el proceso enseñanza-aprendizaje, de una manera intencional y anunciada a los estudiantes. Aunque ocho (8) respondieron que sí aplican escritura en el aula, anotaron que no lo hacen con el propósito anunciado y consciente de escribir para aprender y menos comunicándolo abiertamente en clase. La hipótesis b no se confirmó. Sólo el 2\% de los participantes expresó que no es necesaria la práctica de la escritura en el aula de disciplinas diferentes a las de lectoescritura. En las respuestas del 98\% de los encuestados emergió la variable Transversalidad, para señalar el estatus que debería ocupar la escritura en el aula. Conclusiones: los docentes no están formados para practicar escritura disciplinar en el aula y los currículos no lo consideran. La docencia, generalmente, transmite y reproduce; esto es, se enfoca más en el acto de la enseñanza que en la preocupación porque el estudiante comprenda y apropie.

Palabras clave: Práctica de la escritura, Enseñanza-aprendizaje con la escritura, Escritura y aula universitaria, Transversalidad.

\section{Abstract}

Objective: to establish whether writing is used intentionally as a teachinglearning tool in the classrooms of the Universities in Manizales and to identify writing strategies implemented in the classroom as well as possible curricular guidelines on the matter. Methodology: a survey with closed-ended and open-ended questions and an interview with open-ended questions were administered to 200 professors from five universities of Manizales. The aim was to prove two hypotheses: a) Writing is not intentionally used as a teaching-learning tool in the university classroom b) Professors consider that writing should be taught in the subjects of literacy skills. Results: the first hypothesis was confirmed by 99\% of the answers, 198 out of 200 professors answered that they did not use writing intentionally and explicitly in order to underpin the teaching-learning process in the classroom. Although 8 professors answered that they use writing in the classroom, they stated that they do no not do it with the conscious and explicit purpose of learning and that they do not communicate it openly in class. Hypothesis B was not confirmed. Only $2 \%$ of professors said that it is not necessary to practice writing in the classroom in disciplines different from literacy. The variable Transversality emerged in the answers of 98\% of the professors to indicate the status that writing should have in the classroom. Conclusions: professors are not trained to practice disciplinary writing in the classroom and curricula do not 
consider it. Teaching generally transmits and reproduces, i.e., it focuses more on the act of teaching that it is concerned with the student understanding and appropriation.

Keywords: Writing practice, Writing as a teaching-learning tool, Writing and university classroom, Transversality.

\section{Resumo}

Objetivo: estabelecer se nas salas de aula universitárias de Manizales faz-se uso intencional da escritura como instrumento de ensino-aprendizagem e identificar estratégias para a escritura aplicadas na sala de aula e as possíveis orientações curriculares ao respeito. Metodologia: aplicou-se uma pesquisa (enquete) com perguntas fechadas e abertas e entrevista aberta a 200 professores de cinco universidades de Manizales. Com isso, procurou-se a verificação de duas hipóteses: a) na sala de aula universitária não se usa, intencionalmente, a escritura como instrumento para o ensino-aprendizagem eb) os docentes consideram que o ensino da escritura se deve deixar às matérias de alfabetização. Resultados: a primeira hipótese foi confirmada com um 99\% das respostas. Dos 200 professores participantes, 198 responderam que não usam a escritura na sala de aula para apoiar o processo ensinoaprendizagem, duma maneira intencional e anunciada aos estudantes. Embora oito (8) responderam que efetivamente aplicam a escritura na sala de aula, disseram que não o fazem com o fim anunciado e consciente de escrever para aprender e menos comunicando-o abertamente na aula. A hipótese b não se confirmou. Só $2 \%$ dos participantes expressaram que não é necessária à prática da escritura na sala de aula de disciplinas diferentes às de alfabetização. Nas respostas do 98\% dos entrevistados surgiu a variável Transversalidade, para indicar o status que deveria ocupar a escritura na aula. Conclusões: os docentes não estão formados para praticar escritura disciplinar na sala de aula e os currículos não o consideram. A docência, geralmente, transmite e reproduz; o que significa que, se concentra mais sobre o ato de ensinar que na preocupação porque o estudante compreenda e aproprie.

Palavras-chave: Prática da escritura, Ensino-aprendizagem com a escritura, Escritura e aula universitária, Transversalidade. 


\section{Introducción}

La escritura es el medio de información más antiguo. Un método que permitió generar diversos sistemas alfabéticos no sólo para establecer comunicación, sino también para almacenar y administrar información y conocimiento. Sin embargo, el concepto de escritura que se aborda en este estudio es el de instrumento cognitivo para el aprendizaje, bajo la premisa de que practicar la escritura ayuda a comprender mejor (Cassany, 1993).

Ahora, la escritura no es un fin en sí mismo. Es un medio para aprender o para dar sentido a la existencia. Porque escribir es una manera de existir, de construir y reconstruir el mundo propio. Es la posibilidad de crecimiento continuo y no una simple destreza, ya que la escritura, además de servir de puente para conocer o dar a conocer, también reforma la conciencia; es decir, que la expresión escrita tiene efectos en los pensamientos y sentimientos de quien la emite. En ese sentido, la escritura "descubre el sentido profundo de nuestros actos, de nuestras actitudes y de nuestros sentimientos: la escritura no sólo es un cauce de exteriorización, sino también una vía de interiorización” (Hernández, 2005, p. 37).

Como proceso para la enseñanza-aprendizaje, en el ámbito educativo la escritura se ha establecido como una asignatura, a través de la cual se brinda a los estudiantes los elementos gramaticales y las estrategias de composición escrita. Con esto, se pretende que desarrollen las destrezas comunicativas adecuadas, para un mejor aprovechamiento del quehacer académico-científico. Por tanto, la escritura no es únicamente una materia que "ver" o enseñar. Es un instrumento necesario en todas las disciplinas -en las que, posiblemente, no se esté usandoporque la escritura es un camino para adentrarse en la comprensión disciplinar y evaluar los alcances o límites conceptuales sobre un tema en particular. En general, junto a la lectura, la escritura es condición primordial en el proceso enseñanza-aprendizaje:

La lectura y la escritura son condiciones para mantener las posibilidades individuales de aprendizaje y actualización en los diferentes contextos de desempeño, constituyen vías de acceso a la información y al conocimiento, y contribuyen a la creación y producción de la subjetividad; por tanto, es de asumir que más que temáticas o asignaturas, son componentes transversales de cualquier proceso de formación humana (Cisneros y Vega, 2011, p. 23).

Pensada como instrumento, la escritura es una construcción humana. Es un instrumento inventado en los orígenes humanos. Hoy, constituye un instrumento 
cultural que continúa evolucionando. O instrumento tecnológico, de acuerdo con Ong (1994) quien dice que "la escritura es la invención tecnológica humana más trascendental” (p. 86), que permite desarrollo material y psicológico. Por eso, el uso de este recurso no sólo ha "impulsado y moldeado la actividad intelectual, sino que puede enriquecer la psique humana, desarrollar el espíritu humano, intensificar su vida interior. La escritura es una tecnología interiorizada” (p. 86). Y no sólo tiene un impacto individual, puesto que la práctica de la escritura "soportada en diversos medios y técnicas, tiene un carácter procesual y tiene efectos sobre las poblaciones" (Rojas y Jiménez, 2012, p. 20).

Las razones que impulsan la escritura, son múltiples. Van de lo intelectual, social, cultural a lo estético y lo moral. Esto, precisamente "por los indiscutibles beneficios que se derivan: solaz o placer estético, instrucción, trabajo, aprendizaje, medio para ordenar o clarificar ideas, mnemotecnia y aún algún tipo de terapia” (Niño, 2006, p. 181).

Ahora, cuando se ingresa a la educación superior no se lee y se escribe para leer y escribir, sino para aprender. Como lo asevera Cassany (2009) "Podemos darnos cuenta de que leer y escribir no son fines en sí mismos: uno no lee o escribe para leer o escribir. Al contrario, son formas de lograr objetivos sociales y prácticas culturales más amplios" (p. 24). Como, por ejemplo, el reconocimiento que otorgan los escritos a investigadores y docentes. Éstos, son reconocidos y juzgados por lo que escriben, su identidad académica se fundamenta en sus publicaciones.

Sin embargo, al parecer, (es lo que verificar este estudio) el uso de la escritura en la academia, generalmente, se aplica más como soporte para la transmisión y reproducción de conocimiento, que como instrumento de enseñanza-aprendizaje. Su presencia, la de la escritura, "su forma de aparecer en las tareas diarias de los pregrados y posgrados, [ya] no es tan comprensible", (Cisneros y Vega, 2011, p. 12). Es más, ahora no sólo no parece comprensible el papel de la tarea escrita en el proceso de aprendizaje, sino además poco confiable. Esto, por las posibilidades que tienen los estudiantes para no hacer y de todas maneras entregar escritos, frente a restricciones que tienen los docentes, por ejemplo: demasiados estudiantes y poco tiempo para verificar y pedir la sustentación personalizada de la cantidad de "tareas" escritas o para cotejar los escritos con fines de evitar plagios.

Así, para el estudiante la práctica de la composición escrita se limita a lo aprendido en la materia de lectoescritura y afines en los ciclos básicos e 
intermedios. Y cuando ingresan a la educación superior, los docentes asumen que los estudiantes que llegan a su curso, tienen las competencias para leer o escribir. Pero, quien ingresa a la universidad tiene la expectativa de que aprenderá a leer y a escribir y "espera que el profesor le cuente, le enseñe, lo instruya, le diga qué y cómo leer y escribir, y aún más, lo motive a leer” (Arciniegas y López, 2012 , p. 9) y a escribir. Pero el escenario es otro. Tal como se plantea el ciclo universitario, en síntesis, la situación es que se parte de que los estudiantes ya tienen las habilidades para leer y escribir, mientras que ellos esperan aprender a escribir en la universidad.

El uso de la escritura como instrumento de enseñanza-aprendizaje, sin embargo, implicaría que el profesor asuma el reto no sólo de formar en la disciplina respectiva, sino también ayudar a "desarrollar en sus estudiantes actitudes y competencias que le permitan comprender y problematizar los contenidos propios de su campo disciplinario" (Arciniegas y López, 2000. p. 9). De esta forma, la práctica de la escritura contribuiría a que los estudiantes asuman la responsabilidad de su crecimiento en el aprendizaje, a partir del reconocimiento de la escritura como el instrumento clave del proceso.

\section{La escritura disciplinar en el aula}

La enseñanza en el aula, tradicionalmente, se ha fundamentado en la transmisión de conocimientos. Es un modelo que ofrece pocas oportunidades de usar la escritura para indagar y comprender con apoyo de la experiencia del profesor. Mantiene una forma didáctica que poco, o nada, usa la escritura como instrumento para afianzar conocimiento (al menos esta es la hipótesis sobre la que se adelanta este estudio). Y, en ese sentido, Moyano (2010) afirma que "Se ha hecho escasa, o ninguna mención, a la enseñanza desde una perspectiva funcional, de los usos específicos de recursos lingüísticos y discursivos para la construcción de significado en los diferentes grupos de disciplinas" (p. 467).

Así, en el modelo convencional (magistral) de aula de clase se parte, generalmente, del supuesto de que aprender a escribir se hace en asignaturas de gramática o lectoescritura-premisa que corresponde a la segunda hipótesis del presente estudio-. De ahí que cada docente, desde su disciplina, reclame que los estudiantes llegan a sus cursos sin las competencias para comprender y producir textos.

Estas dificultades constituyen un obstáculo muy difícil de superar en la medida en que se trata de habilidades básicas que permiten la construcción y producción de conocimiento en las diferentes disciplinas. Por otra parte, los propios estudiantes 
entienden éste como un problema a la hora de aprobar determinadas materias y se sienten inseguros para iniciar estudios superiores (García, 2010, p. 285).

Dado el sistema educativo, sin embargo, los cursos avanzan en medio de los vacíos para leer y escribir. Y el aula de clase, sin la práctica de la escritura disciplinar, estaría así contribuyendo a reproducir y a hacer memorizar, más que a estimular nuevos pensamientos. O como lo expresa Carlino (2006) es un modelo "que entiende la docencia como 'decir a los estudiantes lo que sabemos sobre un tema', omite enseñarles uno de nuestros más valiosos saberes: los modos de indagar, de aprender y de pensar en un área de estudio, modos vinculados con las formas de leer y de escribir" (p. 6).

Por eso, Castronovo y Mancovsky (2010) afirman que los docentes no son ajenos a la tarea de fortalecer la habilidad de la escritura en los estudiantes. Argumentan que "la lectura y la escritura son competencias que necesitan desplegar los estudiantes para el logro de sus aprendizajes académicos y es una tarea propia del docente ayudarlos a que lo logren” (p. 819).

Y qué mejor escenario que el aula, para que el docente adicione el valor agregado de incentivar la expresión escrita entre los estudiantes. De contera, con ello aporta al aprendizaje, porque la escritura en el aula se hace sobre contextos significativos, sobre situaciones. Esto implica un proceso cognitivo que obliga al estudiante a organizar y a pensar -y repensar-sus ideas hasta llevarlas a una composición escrita.

La escritura disciplinar ejercitada en el aula, entonces, es la oportunidad para que el estudiante construya o afiance conocimiento con apoyo del profesor. La escritura en el aula permite que interactúen docente y estudiante para "negociar" la certeza de frases o la coherencia de un párrafo; o defender o criticar un concepto en una situación determinada. Este encuentro, que tiene en medio a la escritura, necesariamente contribuye a madurar conocimiento y fortalece la comprensión sobre los contenidos.

Pero, lo normal es que los escritos que presentan los estudiantes son realizados fuera del aula. Esto tiene la desventaja de la ausencia del acompañamiento (por no decir "control”) del proceso. Se le revisa el producto y no el proceso. El primero, tiene más dudosa reputación en cuanto a originalidad. El segundo, el proceso, con acompañamiento en el aula, es la garantía del compromiso del estudiante.

Ahora, en relación con las investigaciones y programas que se han adelantado sobre el uso de la escritura en el aula, desde los años 70 y 80 en Europa y 
América se establecieron estrategias institucionales para integrar la enseñanza en las diferentes cátedras universitarias. Así lo muestra el estudio titulado “¿Qué nos dicen las investigaciones internacionales sobre escritura en la universidad? (Carlino, 2007). En éste, se presenta "un panorama de investigaciones sobre escritura en la universidad, que enfocan cómo se la incluye en la enseñanza en relación con el aprendizaje de las diversas asignaturas" (p. 21).

Esta investigación de Carlino (2007), también da a conocer antecedentes de investigaciones sobre la escritura en Estados Unidos, Australia e Inglaterra, desde la década de los 70. En tales estudios, hubo enfoques sobre el proceso cognitivo de la escritura y sobre la incidencia del contexto en la producción escrita. Y "ha sido más recientemente que la escritura requerida en la universidad empezó a ser entendida como una práctica académica que varía según las culturas institucionales en las que tienen lugar y pasa a ser entendida como constitutiva de los usos discursivos de cada campo del saber" (Carlino, 2007, p. 23).

De acuerdo con lo expuesto por Carlino (2007), surgen dos movimientos pedagógicos que se extienden a varias universidades norteamericanas: "Escribir a través del currículo" en Inglaterra y EE UU. Y más adelante "Escribir en las disciplinas". Ambos movimientos propiciaron la integración "de la enseñanza de la escritura en todas las materias; el primero, como una herramienta para ayudar a pensar los contenidos conceptuales (escribir para aprender) y el segundo, como un modo de enseñar las particularidades discursivas de cada campo del conocimiento (aprender a escribir)" (Carlino, 2007, p. 23).

El resultado principal del estudio en mención es que, de todas formas, "Es infrecuente la enseñanza explícita de los géneros discursivos esperados e insuficiente la orientación dada a los alumnos cuando escriben, en virtud de que se considera natural lo que, en cambio, son modos discursivos propios de cada disciplina" (Carlino, 2007, p. 21). Es decir, los docentes no dan a conocer el género de escritura propio de su disciplina, puesto que asumen como un hecho que los estudiantes ya saben escribir.

En Colombia, en la Universidad del Valle se realizó la investigación $L a$ escritura en el aula universitaria por Arciniegas y López (2012). En ésta, sus autoras muestran la aplicación de un modelo de enseñanza de la escritura basada en el proceso que ayuda al estudiante a tener en cuenta, en el momento de la producción escrita, elementos como el tipo de texto, la audiencia y la conciencia sobre el proceso cognitivo del acto de escribir. 
Nuestro propósito es proponer un programa que, basado en los elementos fundamentales de estas teorías, integre un trabajo consciente y sistemático, orientado al desarrollo de estrategias tanto cognitivas como metacognitivas para la producción textual. Buscamos, de esta manera, que en sus prácticas de lectura y de escritura académicas, los estudiantes asuman un comportamiento estratégico, mediante el que no solamente van a aprender sobre unas temáticas específicas, sino sobre la lengua, sobre los modos específicos de escritura y sobre la regulación del procesos para llegar a así a la construcción de conocimiento (Arciniegas y López 2012, p. 29).

Otro estudio realizado en la Universidad Tecnológica de Pereira titulado "La lectura y la escritura en la universidad. Un investigación diagnóstica" por Cisneros (2006; citada por Cisneros, Olave y Rojas, 2013) muestra que los estudiantes de últimos semestres no tienen habilidades de comprensión lectora y para la composición escrita. Entre otras conclusiones resalta que "la preparación universitaria, estrictamente disciplinar, no impacta en los niveles de relación epistémica de los estudiantes con el texto académico de corte explicativoargumentativo" (p. VII). Es decir, generalmente, el acercamiento a los textos no tiene el acompañamiento adecuado de los docentes, puesto que éstos "se encuentran más atentos a que el estudiante adquiera la información propia de su especialidad, que a la forma como ésta se construye (Cisneros et al., 2013, p. 9).

Cisneros et al. (2013) también refieren que en Colombia algunas universidades han iniciado eventos interinstitucionales en torno a la lectura y la escritura y “ofrecen cursos de capacitación a sus docentes de distintas áreas a fin de que puedan asumir, en mayor o menor medida, la lectura y la escritura en sus procesos docentes" (p. 9).

En la Universidad Sergio Arboleda de Santa Marta, Polo et al. (2011) vienen implementando un programa de enseñanza de la escritura disciplinar en el aula. Exponen los bases teóricas de la escritura como instrumento de aprendizaje y de la posibilidad de la enseñanza de la escritura en la clase disciplinar. Los autores sustentan que a partir de la integración de la escritura en el aula, en cualquier disciplina

Se puede desencadenar un proceso de aprendizaje si adoptamos un conjunto de estrategias procedimentales que conduzcan al alumno a crear mecanismos de desarrollo de ideas. La idea que subyace es: hay que enseñar cómo se desarrollan las ideas temáticas. Sólo cuando el joven ha aprendido a desarrollar párrafos será capaz de escribir textos con cierta coherencia y longitud (Polo, 2011, et al., p. 2). 
En Manizales, Colombia, ciudad en la que se hace este estudio-diagnóstico sobre el uso de la escritura disciplinar en el aula, no se encontraron antecedentes de trabajos similares.

\section{Escritura para el aprendizaje}

La escritura es la actividad intelectual -para muchos "herramienta"- que más avances permite en el proceso de aprendizaje. En realidad, el aprendizaje poco progresa sin la escritura. El acto de escribir es una habilidad cognitiva que está "vinculada con la capacidad para desarrollar un pensamiento abstracto, objetico, lógico y racional” (Cassany, 2009, p. 33), que se requiere en la constante tarea de aprender.

La escritura es una forma de lenguaje, un sistema de signos, y por tanto es una herramienta psicológica. Estas herramientas son medios de actividad interna que introducidas en una función psicológica, como el pensamiento por ejemplo, la transforman cualitativamente. Por su carácter de herramienta psicológica, la adquisición de la lengua Escitia modifica la estructura de los procesos cognitivos, llevando a los seres humanos del razonamiento práctico-situacional hacia el pensamiento teórico-conceptual y narrativo, lo que implica la aparición de nuevas y más elevadas formas de pensamiento (Valery, 2000, p. 40).

La escritura es un proceso cognitivo que implica la posibilidad de descubrir y aclarar lo que se quiere comunicar. Y más allá de un medio de transmisión, la escritura esconde un potencial epistémico que se revela a quien se aventura. Porque las buenas ideas surgen gradualmente. Es decir, trasiegan un camino de varias reescrituras hasta que maduran. De ahí que la composición escrita sea esencial en la construcción de conocimiento y en el desempeño académico. Esto, porque la escritura apoya el razonamiento:

La escritura es adecuada para aprender a razonar por varios motivos. Por un lado, exponer una idea implica primero hacerla tuya. Por otro lado, la escritura es exigente y obliga a los alumnos a buscar la palabra adecuada, a no dejar agujeros en las líneas de razonamiento, a buscar ejemplos apropiados (Miró, s. f., p. 2).

Por eso, la escritura es instrumento de aprendizaje. "Es la herramienta privilegiada, inigualable, para organizar nuestros conocimientos y conceptuarlos mejor" (Boyson-Bardies, 2007, p. 196). Escribir es el apoyo del pensamiento. Ayuda a ordenar y consolidar conocimiento y a razonar más críticamente. Esto, porque 
(...) la composición de un tex to implica la ejecución de una serie de operaciones que permiten pasar de un estado de conocimiento a otro. Así, en el espacio del contenido, alrededor del tópico de la composición, el escrito determina su saber, la información previa y sus creencias al respecto y decide, entonces, lo que aún necesita investigar sobre el asunto para cumplir con los requisitos establecidos" (Arciniegas y López, 2012, p. 17).

En el acto de escribir, se descubren "los puntos débiles que deben reforzarse, puntos fuertes que hay que explotar. El trabajo que vayas haciendo y el conocimiento que saques de él dará más fuerza a tu escritura, hará tus frases más precisas, tus razonamientos serán más potentes, mejorará su estructura” (Miró, 2011, p. 11). Porque probado está que la escritura es "un instrumento epistemológico de aprendizaje. Escribiendo se aprende y podemos usar la escritura para comprender mejor cualquier tema” (Cassany, 1993, p. 32).

En la práctica de la composición escrita, surgen preguntas que no se habían contemplado respecto a un tema. Y como el proceso de la escritura es un clarificador de los pensamientos, en él podrían hallarse respuestas inesperadas. Es decir, se adquiere conocimiento. "Escribir es una estrategia para fortalecer el conocimiento disciplinar, científico, cultural y personal, puesto que es un instrumento de explicación, aprendizaje y cambio” (Sánchez, 2011, p. 27).

Escribir es preguntarse; es reflexionar sobre el propio saber, ya que "Se escribe con toda la experiencia acumulada” (Creme y Lea, 200, p. 17). La redacción ayuda a ordenar los conceptos, puesto que en la ejecución cognitiva del acto de redactar, ocurre el momento-lugar en el que se consolidan o toman forma las ideas. "Escribir es, sobre todo, una manera de aprender" (Creme y Lea, 2000, p. 22).

Todo lo anterior resalta la importancia de verificar si en el aula universitaria se practica la composición escrita para fortalecer el proceso de enseñanzaaprendizaje disciplinar. Esto, con el objeto de identificar experiencias exitosas de estrategias en este campo y determinar si se realizan en el aula de manera intencional y anunciada a los estudiantes. Además, se busca revisar si existen indicaciones curriculares para vincular la escritura como práctica disciplinar en el aula y si los docentes consideran que esto sea necesario o que tal ejercicio se deba relegar a asignaturas específicas. Para ello, se plantean dos hipótesis: a) en el aula universitaria no se usa, intencionalmente, la escritura como instrumento para la enseñanza-aprendizaje y b) los docentes consideran que la enseñanza de la escritura se debe dejar a las materias de lectoescritura. 
Este estudio, se justifica porque sus resultados podrían aportar a los agentes de decisión de las instituciones educativas e, incluso, de los entes que establecen las políticas públicas de la educación. También, por los estudios que se pueden derivar de éste en relación con los procesos educativos o la elaboración de la propuesta de un modelo de enseñanza de escritura disciplinar en el aula.

\section{Metodología}

El camino metódico que siguió este estudio fue la búsqueda y análisis de información para confirmar o rechazar las dos hipótesis planteadas en la Introducción. Esta fuente de información provino de los docentes universitarios que orientaban asignaturas distintas a la asignatura de lectoescritura o talleres de escritura.

Así, la muestra para este estudio se conformó con 200 profesores de cinco universidades de Manizales ${ }^{1}$; dos públicas: Universidad de Caldas (45) y la Universidad Nacional, sede Manizales (40); y tres privadas: Universidad de Manizales (45), Autónoma de Manizales (35) y Universidad Católica (35). Los docentes que participaron en este estudio, se seleccionaron aleatoria, o incidentalmente; es decir, fueron encuentros casuales que surgieron en los recorridos que hizo el responsable de este trabajo en las universidades. La única salvedad, era que el docente no orientara materias de gramática o lectoescritura.

La muestra, entonces, abarcó las siguientes asignaturas: Matemáticas, Geología, Medicina, Agronomía, Biología, Ingenierías, Sistemas, Arquitectura, Administración de Empresas, Economía, Política, Ética, Comunicación Social y Periodismo.

Para recolectar la información, se aplicó una encuesta y una entrevista abierta, espontánea. En la encuesta se plantearon dos preguntas, a partir de las cuales se confirmaban o rechazaban las dos hipótesis. Cada una de las cuales pedía una respuesta cerrada y una abierta.

Además con la entrevista, muchos de los participantes enriquecieron las respuestas con la narración de sus vivencias y actitudes el aula de clase,

1. Como se explicará adelante, para este estudio no fue relevante la clasificación de los docentes de acuerdo con su tipo de contrato (de planta, ocasional, catedrático), sexo, edad, formación. En la fecha, el número total de docentes de las universidades en las que se hizo la encuesta y la entrevista fue 2.664: Universidad de Caldas (968); Universidad Nacional, sede Manizales (363); Universidad de Manizales (668); Universidad Autónoma de Manizales (415) y Universidad Católica de Manizales (250). 
relacionadas con procesos de escritura. Las dos cuestiones que se plantearon en la encuesta, fueron: 1) En su aula de clase ¿utiliza intencionalmente la escritura como instrumento de aprendizaje?² $\mathrm{SI}(\quad$ ) NO ( ) ¿Cómo?; y 2$)$ ¿Se debe dejar la enseñanza de la escritura únicamente a la materia de lectoescritura? SI ( ) NO ( ) ¿Por qué?

Estas dos preguntas y la entrevista, estuvieron dirigidas sólo a docentes universitarios. La muestra para este estudio se conformó con 200 profesores de cinco universidades de Manizales $^{3}$; dos públicas: Universidad de Caldas (45) y la Universidad Nacional, sede Manizales (40); y tres privadas: Universidad de Manizales (45), Autónoma de Manizales (35) y Universidad Católica (35). En el momento, todos los docentes seleccionados orientaban materias distintas a la asignatura de lectoescritura o talleres de escritura.

La muestra cubrió las siguientes asignaturas: Matemáticas, Geología, Medicina, Agronomía, Biología, Ingenierías, Sistemas, Arquitectura, Administración de Empresas, Economía, Política, Ética, Comunicación Social y Periodismo.

Con las estadísticas provenientes de las respuestas a la pregunta cerrada, se emitieron los resultados en cifras y porcentajes y se hizo el análisis interpretativo y de discusión con resultados y teorías de otros estudios. Y con las respuestas y opiniones sobre las preguntas abiertas, se hizo una lectura y relectura cuidadosa para determinar, en su conjunto, la emergencia de posibles categorías.

\section{Resultados}

Los hallazgos se presentan alrededor de un eje articulador que descansa en las dos hipótesis. Es decir, estos dos aspectos: a) la determinación del uso de la escritura en el aula, la evidencia de posibles experiencias y directrices curriculares al respecto y b) la opinión de los docentes en el sentido de qué lugar debe ocupar la escritura en el currículo académico superior. En cada uno

2. La encuesta explicaba que usar intencionalmente la escritura como instrumento de aprendizaje en el aula, en los términos de este estudio, implica que el docente lo anuncie y aclare entre los estudiantes y que todos asuman la práctica como tal.

3. Como se explicará adelante, para este estudio no fue relevante la clasificación de los docentes de acuerdo con su tipo de contrato (de planta, ocasional, catedrático), sexo, edad, formación. En la fecha, el número total de docentes de las universidades en las que se hizo la encuesta y la entrevista fue 2.664: Universidad de Caldas (968); Universidad Nacional, sede Manizales (363); Universidad de Manizales (668); Universidad Autónoma de Manizales (415) y Universidad Católica de Manizales (250). 
de estos dos tópicos se presentan los resultados y se interpretan comparando o discutiendo con estudios similares para dar significado en el contexto actual. Se evidencia, además, la variable que emerge de las respuestas a la encuesta y de los testimonios de los docentes participantes.

El principal resultado, entonces, es que se confirmó la primera hipótesis al evidenciarse que en el aula universitaria de Manizales no se practica, de manera intencionada, la escritura para fortalecer el proceso enseñanza- aprendizaje. Frente a la pregunta 1 sobre si usa la escritura en clase para fortalecer la enseñanza-aprendizaje, de manera intencionada y anunciada a los estudiantes, 198 de los 200 docentes encuestados respondieron que no. Es un resultado trivial -se podría juzgar- de una hipótesis que más parece un hecho natural ya dado ${ }^{4}$ en la educación superior. Lo que importa, sin embargo, es lo que significa en el contexto académico, además de las lecturas pedagógicas, didácticas, curriculares y políticas, que podrían emerger al llevar este asunto a la palestra académica.

Ahora, aunque seis (8) de los 200 profesores encuestados, un $4 \%$, manifestaron practicar, ocasionalmente, escritura en el aula, expresaron que no lo hacen con la intención consciente -mucho menos anunciada a los estudiantes- de aplicar una estrategia epistémica mediante la escritura. Significa esto, que en el sistema educativo y en las instancias curriculares están ausentes las iniciativas formales para que la escritura tenga una función más relevante en el aula de cada disciplina. Significa también que, de pronto, la educación superior sigue privilegiando la impartición (transmisión) de contenidos: parece más importante la carga de información a transmitir que los momentos para digerir algo de ella. "Yo no puedo variar el programa temático; practicar la escritura como lo plantea la pregunta, implicaría dejar de entregar el contenido pactado y eso no lo puedo hacer" (Docente universitario).

En consecuencia, este hallazgo confirma que, con las debidas excepciones, docentes e instituciones no han considerado incluir en el aula la práctica de

\footnotetext{
4. Que no se use la escritura en el aula, en las condiciones en que lo plantea este estudio, es aparentemente obvio. No obstante, uno de los aportes de la academia, y la ciencia, es la consideración teórica, sistemática o científica, sobre fenómenos que aparecen obvios en la realidad. Es la manera como se reflexiona sobre su proceso o se abordan sus causas, teorías, consecuencias o impacto, lo que justifica estos trabajos. Por eso, no importa, en este caso, el tamaño de la muestra. No sólo 200, sino que, seguramente, la mayoría, tal vez el $99 \%$, de los 2664 docentes universitarios que conforman el total de la población objeto (el universo, para este estudio) responderían lo mismo. Lo que busca este estudio, entonces, no es tanto sobre-evidenciar que existe tal fenómeno, sino reflexionar sobre él desde, y en torno a, las investigaciones académicas que lo han abordado.
} 
la escritura para que los estudiantes tengan la oportunidad de comprender y aprender mejor, aprovechando la presencia del profesor. Frente a este punto, otro estudio sobre escritura en el aula realizado por Hernández et al. (2003) plantea que "La escuela debe ser el espacio donde el alumno ponga en práctica el ejercicio de la escritura, ¿̇y qué mejor que hacerlo contando sus experiencias, dentro de la cotidianidad del aula de clase? Si hay que desarrollar habilidad para escribir, es el ámbito educativo el llamado a brindar este espacio y se debe hacer de manera deliberada y sistemática, y no como actividades aisladas dentro del aula de clase (p. 4). Sin embargo, como lo muestra este estudio, esta no es ni una política ni una estrategia oficialmente declarada en la educación superior.

Algunas de las razones que expusieron los profesores participantes en este estudio, para que se presente este fenómeno, son:

- "A los docentes no se nos capacita para usar la escritura disciplinar como herramienta de aprendizaje" (Docente universitario).

- "La cantidad de estudiantes asignados al aula, a veces 40 o 50, hace imposible actividades de composición donde el docente pueda aportar algo, en forma más personal" (Docente universitario).

- "No hay en la institución una directriz curricular para vincular la escritura en el aula disciplinar" (Docente universitario).

Ahora, entre los ocho encuestados que respondieron que sí hacen uso de la escritura en el aula, aunque no de manera intencional o anunciada, sustentaron algunas estrategias creadas por ellos mismos. Por ejemplo: "La mesa redonda al revés". Esta actividad consiste en ubicar los estudiantes sentados en mesa redonda, pero dándose la espalda. No se miran. El docente indica que uno de los estudiantes escriba en una hoja en blanco un título. Este título debe provenir del tema que el docente acaba de exponer (y en algunos casos con lecturas previas entregadas por el docente).

Cuando el estudiante señalado escribe el título, entrega la hoja al que sigue a su derecha. Éste, inicia un párrafo, pero escribe máximo dos oraciones. Quien continúa aporta otras dos proposiciones que guarden coherencia con lo que escribió el anterior. Y un tercero, finaliza el párrafo. El estudiante que siga en turno, inicia otro párrafo. Cuando vuelve al primer estudiante, éste recibe el escrito completo y lo lee para todos.

La mesa redonda se voltea y los estudiantes quedan de frente para escuchar la lectura del escrito elaborado por todos. Se abre el diálogo para discutir cada 
frase o párrafo, si es necesario. Al final, el profesor se queda con el escrito y reta a cada estudiante a que reescriba el escrito a su manera.

El docente de esta propuesta de la “mesa redonda al revés”, declaró que

"Es un ejercicio que aplico sacrificando algo del contenido que hay que impartir; por eso, no se puede hacer mucho. Pero es una experiencia que genera una expectativa interesante entre los estudiantes. La mayoría reconoce que es un ejercicio de repaso, de aprendizaje o de consolidación del conocimiento sobre el tema propuesto en las clases" (Docente universitario).

Otra forma encontrada de practicar escritura en el aula (sin proponerla abiertamente como una táctica para aprender mejor) es la de reescribir párrafos de un texto dado. El escrito corto es entregado a cada estudiante (o el docente solicita que cada estudiante lleve uno a clase) y a partir de éste, se debe redactar un párrafo de cada uno de los que tiene el texto. En síntesis, se intenta reescribir lo que dice el autor con sus palabras, pero intentando poner algunas propias; es decir, un parafraseo. La mayoría de las veces el docente tiene que estar presto a ayudar a aclarar o a construir o a saber qué estudiante le podría colaborar a otro. "El número de estudiantes en el aula es una condición que restringe la práctica de este ejercicio. Pero ayuda a que ellos tomen confianza para abordar textos académicos" (Docente universitario).

Otro profesor entrevistado indicó que "La composición de oraciones cortas, o proposiciones, en la misma clase, ayuda a que los estudiantes aclaren dudas. A algunos les ayudo cuando no avanzan. Estas proposiciones les sirven como resumen del tema visto" (Docente universitario).

En estos ejercicios declarados por algunos de los participantes, principalmente el primero y el último, hacen parte del uso de la escritura para el aprendizaje. Casos que no se tomaron como una respuesta positiva a la primera pregunta de este estudio, puesto que no cumplían la condición de realizarse con el propósito consciente del profesor de usar la escritura para el aprendizaje y anunciarlo, así, abiertamente a los estudiantes. Y además, porque, como lo muestra el estudio de López y Cisneros (s.f.) en estas situaciones así planteadas los estudiantes elaboran un texto escrito en una situación aún de evaluación, es decir, no pensando en construir con autonomía, sino "asumiendo la escritura como una tarea para ser evaluada por el profesor, en la que lo importante es mostrarle que puede responder a lo que le pide” (p.12). 
En relación con la segunda hipótesis (los docentes consideran que la enseñanza de la escritura se debe dejar a las materias de lectoescritura) no se cumplió. De los 200 docentes encuestados sólo cuatro (4), un 2\%, expresaron que la práctica de la escritura en el aula, se debe dejar a las asignaturas propias de la lectoescritura y la gramática. En las respuestas de los demás, el 98\%, surgió la variable Transversalidad, para señalar cómo debe ser la composición escrita en el transcurso académico de los estudiantes.

Con la Transversalidad, ese $98 \%$ de los docentes encuestados muestra que tiene claridad en que la escritura es un componente esencial, y no secundario, en la formación de toda disciplina. Y están de acuerdo en que la práctica de la escritura debe atravesar las aulas de las diversas disciplinas: "Es decir, que en los planes de estudio se contemple como una exigencia que el profesor incluya momentos en el aula, para actividades de fortalecimiento del aprendizaje temático" (Docente universitario). Las respuestas también coincidieron en señalar que para establecer un programa sistemático de escritura disciplinar en el aula, esto es para lograr Transversalidad, "las iniciativas tienen que partir del Ministerio y de las instituciones, para que los docentes finalmente asuman este acto con todo el compromiso" (Docente universitario).

Sin embargo, esa transversalidad a la que aspira la mayoría de los entrevistados en este estudio, para algunos resulta ser "una utopía, ya que el sistema educativo está diseñado más para informar que para formar, por aquello de la cobertura" (Docente universitario).

\section{Conclusiones}

En la práctica docente escasea la vinculación de la escritura como apoyo para la comprensión de contenidos disciplinares. El enfoque que el sistema educativo universitario, parece privilegiar, es el de transmitir y reproducir; es decir, está más enfocado a la enseñanza que al aprendizaje que logre el estudiante. Así, "la escuela pierde su norte cuando se preocupa más por la enseñanza que por el aprendizaje (...) y es evaluada más por sus efectos cuantitativos, estadísticos, pero no desde la esencia de su pedagogía” (Zuluaga, 2008, p. 15).

Al igual que en el trabajo de Hernández et al. (2003) el presente estudio defiende que la participación más comprometida del estudiante en el aula de clase a través de la escritura, ayuda a que los estudiantes logren participar o desenvolverse mejor en la sociedad. Esto, porque la práctica de la escritura es 
un ejercicio para pensar, repensar y concretar ideas y visiones más elaboradas y conscientes que, finalmente, fortalecen las competencias lingüísticas. En este escenario, un programa de escritura en el aula, podría apuntar a que los estudiantes asuman la escritura como un proceso auto-formativo de construcción de sentidos disciplinares.

Una práctica de composición escrita en el aula con el propósito de fortalecer la enseñanza-aprendizaje, permite al estudiante, no sólo una mayor comprensión sobre la diferencia entre decir y transformar o construir conocimiento, sino también conocer controlar y evaluar su propio proceso de composición escrita.

Otra de las conclusiones de este estudio, es que los docentes no están formados para practicar escritura disciplinar en el aula y los currículos tampoco lo consideran. La consecuencia es que la práctica de la escritura queda relegada, sistemáticamente, a las materias de lectoescritura o afines. Sin embargo, aprovechando que la mayoría de los docentes, como lo demuestra este estudio, son conscientes de que se debería pensar en vincular la práctica de la escritura en todas las disciplinas de una manera transversal, se debería establecer como política pública de la educación. "En otras palabas, establecer los tempos curriculares necesarios para el desarrollo de estos aprendizajes y permitir la práctica favoreciendo el desarrollo adecuado de lecturas y escrituras" Cisneros y Vega, 2011, p. 53).

Mediante la escritura disciplinar en el aula se genera una interrelación epistémica más directa del docente con los estudiantes: dialoga la experiencia y conocimiento del profesor con la voluntad dispuesta del estudiante, mediados por la escritura. Son momentos cruciales que ocasionan oportunidades de aprendizaje. No importa la cantidad: un párrafo, o dos o tres; se trata de animar una creación personal que vaya más allá de la redacción frívola y superficial acostumbrada, para la cual basta una información sugerida o impuesta, que ni siquiera es necesario comprender (Niño, 2006).

Con la práctica de la escritura disciplinar, el aula se convierte en escenario para construir conocimiento y no tanto para transmitirlo. Esto, para que "a las aulas de la universidad se vaya más a colegir y borrar que a transcribir; en otras palabras, a contrastar la acción y el pensamiento propio con lo que otros han hecho y dicho a fin de sacar conclusiones que emborronen las certezas" (Cisneros y Vega, 20011, p. 18). 
Lo anterior, "Implica reconocer que el estudiante necesita una continuidad formativa al momento de ingresar a la educación superior, fase siguiente de un desarrollo cuyo antecedente le solicitaba otro tipo de lecturas..." (Cisneros, Olave y Rojas, 2013, p. 3) y de habilidades para la expresión escrita.

Y para lograr tal reconocimiento, docentes e instituciones deberían comenzar por olvidar el supuesto de que los estudiantes que llegan a la educación superior, vienen preparados para abordar, leer y escribir, textos académicos. Esto, porque tal asunción ha ocasionado que no se tenga una clara consideración sobre la necesidad de una propedéutica en la práctica de la escritura disciplinar en el aula, tanto para los estudiantes como para los docentes.

\section{Referencias}

Arciniegas, E. y López, E. (2012). La escritura en el aula universitaria. Estrategias para su regulación. Cali, Colombia: Universidad del Valle.

Boyson-Bardies, B. (2007). ¿Qué es el lenguaje? México: Fondo de Cultura Económica.

Carlino, P. (2006). Escribir, leer y aprender en la universidad. Una introducción a la alfabetización académica. España: Fondo de Cultura Económica.

Carlino, P. (2007). ¿Qué nos dicen las investigaciones sobre la escritura en la universidad? Cuadernos de psicopedagogía (4), 21-40. Universidad Pedagógica y Tecnológica de Colombia, Tunja. Recuperado de: http:// www.fvet.uba.ar/postgrado/especialidad/blb/Carlino_Que_nos_dicen_ las_investigaciones_internacionales_Cuad_Pedag_07.pdf.

Cassany, D. (Comp.) (2009). Para ser letrados. Voces y miradas sobre la lectura. España: Paidós.

Cassany, D. (1993). La cocina de la escritura. Barcelona: Editorial Anagrama

Castronovo, A. y Mancovsky, V. (2010). La lectura y la escritura como procesos centrales de los aprendizajes disciplinares: la inclusión y la pertenencia a una comunidad de lectura específica. En A. Vásquez, M. C. Novo, I. Jakob y L. Peliiza (Comps.). Lectura, escritura y aprendizaje disciplinar (pp. 819-828). Argentina: Universidad Nacional Río Cuarto. Recuperado de: http://www.unrc.edu.ar/ unrc/comunicacion/editorial/repositorio/978-987-688-007-7.pdf 
Cisneros, M. y Vega, V. (2011). En busca de la calidad educativa a partir de procesos de lectura y escritura. Pereira: Universidad Tecnológica de Pereira.

Cisneros, Olave y Rojas (2013). Alfabetización académica y lectura inferencial. Bogotá, Colombia: ECOE Ediciones

Creme, P. y Lea M. (2000). Escribir en la Universidad. Barcelona: Gedisa Editorial

García, R. (2010). Leer y escribir para aprender o cómo se lee y escribe en el aula de la escuela secundaria. En A. Vásquez et al. (Comps.). Lectura, escritura y aprendizaje disciplinar (pp. 285-292). Facultad de Psicología, Universidad Nacional de Mar del Plata. Recuperado de: http:/ /www.unrc.edu.ar/unrc/ comunicacion/editorial/repositorio/978-987-688-007-7.pdf.

Hernández, J. A. (2005). El arte de escribir. España: Ariel.

Hernández, M. L., Castañeda, L. M., Gómez, B. E. y Domínguez, M. S. (2003). Lectoescritura en el aula. Universidad de Antioquia. Recuperado de: http:// www.udea.edu.co/portal/page/portal/bibliotecaSedesDependencias/ unidades Academicas/FacultadEducacion/PresenciaInstitucional/ Regionales/NodoLenguaje/Publicaciones/lecto_escritura_alma.pdf

López, G. S. y Arciniegas, E. (s. f.). Regulación de la escritura en el aula universitaria: la evaluación pedagógica. Recuperado de: http:// ascun-redlees.org/2012/wp-content/uploads/2012/10/23Ponencia20JUNIO2012\%C3\%BAltima.pdf

Niño, V. M. (2006). Competencias en la comunicación. Hacia las prácticas del discurso. Bogotá: Ecoe Ediciones

Miró, J. (s. f.). Aprendizaje a través de la escritura. Recuperado de: http://bioinfo. uib.es/ joemiro/LibArtic/aatdleT.pdf

Moyano, E. (2010). Escritura académica a lo largo de la carrera: Un programa institucional. Revista Signos 43(74), 465-488. Recuperado de: http://www. redalyc.org/articulo.oa?id $=157016715004$

Ong, W. J. (1994). Oralidad y escritura. Tecnología de las palabras. Colombia: Fondo de Cultura Económica. 
Ortega, A. y Torres, S. (1994). Consideraciones metodológicas de la producción escrita en el aula de español como lengua extranjera. ASELE. Actas (IV), 301-312. Centro Virtual Cervantes. Recuperado de: http://cvc.cervantes. es/ensenanza/biblioteca_ele/asele/pdf/04/04_0301.pdf

Polo, N., Bustamante, A. L. y Avendaño, A. (2011). Una experiencia con escritura predisciplinar. Universidad Sergio Arboleda de Santa Marta. Recuperado de: http://es.scribd.com/doc/61936621/Una-ExperienciaCon-Escritura-Unesco

Rojas, G. y Jiménez, H. (diciembre, 2012). La educación superior desde la lectura y la escritura. Amaz. Inves. 3(1), 19-35. Recuperado de: https://www.google.com.co/?gws_rd=cr\&ei=_glTUryIGZPE9gSs6IHABw\#q=Los+estudios + sobre+escritura + en+la + universidad $\% 3 \mathrm{~A}+$ Rese $\%$ C $3 \% \mathrm{~B} 1 \mathrm{a}+$ para+una $+1 \%$ C3\%ADnea+de+investigaci\%C3\%B3n+incipiente

Sánchez, A. (2011). Manual de redacción académica e investigativa: cómo escribir, evaluar y publicar artículos. Medellín: Fundación Universitaria Católica del Norte.

Valery, O. (2000). Reflexiones sobre la escritura a partir de Vigostky. Revista Educere 3(9), 38-43. Recuperado de: http://www.redalyc.org/articulo. oa? id $=35630908$

Zuluaga, R. D. (2008). 300 estrategias de animación a la lectura. Manizales, Colombia: Editorial Manigraf. 